\title{
Interactive comment on "The Beijing Climate Center Climate System Model (BCC-CSM): Main Progress from CMIP5 to CMIP6" by Tongwen Wu et al.
}

\section{Anonymous Referee \#2}

Received and published: 17 January 2019

This is a description paper of BCC-CSM2-MR. The upgraded schemes are well described, while evaluations for internal variability and long-term trends are insufficient. Authors emphasized improvements in several aspects. Some of them (e.g., representation of QBO) are remarkable improvements but some others are not very convincing. Major revisions and some additional analyses are required as follows:

Major comments:

Printer-friendly version

1 The paper lacks the information of ensemble size of each historical runs. The following two comments are related to this issue:

Discussion paper

1.1 Is the global warming trend of CMIP6 model shown in Fig. 10 the ensemble-mean 
value? What is the gray shading? Maximums/minimums of CMIP5? If the ensemble size of CMIP6 is only one member, you cannot deny the possibility that the simulated slowdown after 1998 happened by chance due to natural variability. On the other hand, if it is ensemble-mean value, then the slowdown after 1998 implies it is the result of external forcing. In the latter case, you need to discuss whether the difference in the trends between CMIP5 and CMIP6 is due to the difference in external forcing or the model update.

1.2 Is the improvement of MJO shown in Fig. 7 robust? A correlation coefficient depends on a sample size. Are the sample sizes (the total numbers of years) you used for the analyses of the observation, BCC-CLM1.1m, and BCC-CLM2-MR comparable? If the improvement is true, what do you think is a factor improving the representation of MJO? New convection scheme?

2 It seems that the results in Fig. 8 and Fig. 11 are inconsistent. Is it due to the difference between the reference datasets (NSIDC, ECMWF forecasts, and Hadley Centre Sea Ice)? Please add some explanation to figure out it and the reason why you used those datasets.

3 Section 4.2: Please discuss the SST cold bias in the equatorial Pacific and the double ITCZ problem. Evaluations for the subsurface ocean (temperature, zonal currents) are also required.

4 Section 4.6: There is a large seasonal cycle in the East Asian climate. Analyses should be done for each season, especially for JJA and DJF.

5 Comparison of ENSO representation (NINO3.4 time series, amplitude, spatial pattern etc.) is necessary to evaluate the model performance.

Minor issues:

6 L19: models -> model's

7 L27-28 "Compared to BCC CMIP5 models, BCC CMIP6 models show...": The ex-

Discussion paper

Printer-friendly version 
pression "models" is inappropriate. This paper compared only one model for each (BCC-CSM1.1m for CMIP5 and BCC-CSM2-MR for CMIP6).

8 L41: More -> more

9 L53: The full name of CMIP5 and Taylor et al. (2012) should appear at L42.

10 L59: Please comment on Section 5 and 6.

11 L64: Please add the full name for NCAR.

12 L64: Coupler -> coupler

13 L65-66: Are tuning parameters also the exactly same between the two models?

14 L79: Please add the level of the top layer.

15 L240: What is the CEVSA model?

16 L243: Table 2 -> Table 3

17 L362: 2014 -> 2005? The end of the CMIP5 historical run is 2005.

18 L371 "1986-": "1985-" in the caption of Fig. 1

19 L374-376: In the mid latitude, discrepancies in the two observations are also large. You cannot discuss the difference between the two model.

20 L396: Precipitation data diagnosed in reanalysis is not necessarily correct. You had better use the observation-based dataset.

21 L406: Figure 4s -> Figure 4

22 L409: Were there any reason you used the two different reanalysis datasets (NCEP and ERA-interim) for each analysis?

Printer-friendly version

23 L437 "forcing is less adequate": What forcing?

Discussion paper

24 L452: improvementcompared -> improvement compared 
25 L478-481: Is the lower and deeper NADW better? Please show the observation based values.

26 L486 "from 60S to 60N": Why did not you use the average from 90S to $90 \mathrm{~N}$ ?

27 L491, L500: HadCRU -> HadCRUT4

28 L503: Figure 2c -> Figure 10?

Interactive

29 L529: abruptCO2 -> abrupt $\mathrm{CO} 2$

30 L535 "the TCR of the new version model BCC-CSM2-MR is lower than BCCCSM1.1m,": lower -> higher? It is inconsistent with L527-528.

31 L566-567: Are there any impact of the upgraded land surface scheme on the improvement of the rainfall diurnal cycle?

32 L616-620: Please see 31.

33 Table 3 "(Guenther et al., 2012)": Please check the font.

34 Table 4: What is the value of the net energy at TOA for OBS? 0.81 ?

35 Table 4, Notes, "1981-2014": 1981-2005 for BCC-CSM1.1m? Why are the periods slightly different among the figures or tables (1985-2005 for Fig. 1, 1986-2005 for Fig. 2-3 and 5, 1980-2005 for Fig. 4, 8-9, 13)?

36 Figure $7 \mathrm{~d}$-f: Please show the range of longitude used for the average to obtain the latitude-time section.

37 Figure 14: The result of BCC-CSM1.1m (BCC-CSM2-MR) should be in the bottom (middle) as same as in the other figures.

Printer-friendly version

Interactive comment on Geosci. Model Dev. Discuss., https://doi.org/10.5194/gmd-2018-254, 2018. 